\title{
Charge-Transfer Selectivity and Quantum Interference in Real-Time Electron Dynamics: Gaining Insights from Time-Dependent Configuration Interaction Simulations
}

\author{
Raghunathan Ramakrishnan ${ }^{1 *}$ \\ ${ }^{1}$ Tata Institute of Fundamental Research, Centre for Interdisciplinary Sciences, Hyderabad 500107, India
}

(Dated: March 9, 2020)

\begin{abstract}
Many-electron wavepacket dynamics based on time-dependent configuration interaction (TDCI) is a numerically rigorous approach to quantitatively model electron-transfer across molecular junctions. TDCI simulations of cyanobenzene thiolates - para- and meta-linked to an acceptor gold atom-show donor states conjugating with the benzene $\pi$-network to allow better through-molecule electron migration in the para isomer compared to the meta counterpart. For dynamics involving non-conjugating states, we find electron-injection to stem exclusively from distance-dependent nonresonant quantum mechanical tunneling, in which case the meta isomer exhibits better dynamics. Computed trend in donor-to-acceptor net-electron transfer through differently linked azulene bridges agrees with the trend seen in low-bias conductivity measurements. Disruption of $\pi$-conjugation has been shown to be the cause of diminished electron-injection through the 1,3-azulene, a pathological case for graph-based diagnosis of destructive quantum interference. Furthermore, we demonstrate quantum interference of many-electron wavefunctions to drive para- vs. meta- selectivity in the coherent evolution of superposed $\pi(\mathrm{CN})$ - and $\sigma(\mathrm{NC}-\mathrm{C})$-type wavepackets. Analyses reveal that in the para-linked benzene, $\sigma$ and $\pi$ MOs localized at the donor terminal are in-phase leading to constructive interference of electron density distribution while phase-flip of one of the MOs in the meta isomer results in destructive interference. These findings suggest that a priori detection of orbital phase-flip and quantum coherence conditions can aid in molecular device design strategies.
\end{abstract}

\section{INTRODUCTION}

First-principles understanding of why, how and how much electric current flows across a given molecule holds the key to unlock challenges in designing electronic circuits of sub-nanometer dimensions with atomistic precision [1 8]. Pioneering efforts in the design of scanning tunneling microscope-based break junction (STMBJ) experiments have made it feasible to accurately determine the through-molecule conductance, $G=I / V$, of a device at vanishing bias-voltage when the molecular electronic structure is least perturbed [9]. A histogram of $G$ is made by recurrently forming and breaking the contacts between molecules and the STM tip where spikes for values less than the quantum of conductance $G_{0}=$ $2 e^{2} / h \approx 7.75 \times 10^{-5} \mathrm{~S}$ indicate flow of current through single molecule junctions [10]. As for first-principles modeling of quantum conductance, the standard approach is the Landauer formalism for coherent transport - valid for short junctions at low temperatures - wherein electrons flow across molecules through conduction channels that are related to the molecular orbitals (MOs) [11. At zero bias voltage, conductance is calculated as $G(E, V)=$ $G_{0} \sum_{i, j} T_{i, j}(E, V)$, where $T_{i, j}$ is the probability that a charge carrier coming from a terminal in transverse channel $i$ will be transmitted to another terminal in channel $j$. This formalism based on the nonequilibrium Green's function (NEGF) method[12] has found wide applicability when used with MOs modeled at various quantum

\footnotetext{
* ramakrishnan@tifrh.res.in
}

chemistry levels of theory ranging from the empirical Hückel MO (HMO) model Hamiltonian 13 to the KohnSham density-functional theory (KS-DFT) [14]. For prototypical systems, both NEGF-HMO and NEGF-DFT methods have shown to give qualitatively similar transmission spectra, while the former accounts only for $\pi$ tunneling, the latter approach not only captures tunneling via all the MOs but also provides quantitatively accurate treatment of electronic interaction [15].

Quantum interference (QI) is an experimentally quantifiable effect stemming from the phase differences of the current flowing through multiple pathways within a molecular junction[16 18]. Interest in QI had its beginnings from observations on a mesoscale metal ring $(\approx \mu \mathrm{m}$ in diameter) where the resistance as a function of applied magnetic field displayed oscillations characteristic of the Aharonov-Bohm effect 19]. One of the prime physical factors that destroy the QI effects is inelastic scattering during conduction. Minimizing such scattering effects requires the loop dimension to be of the size of the benzene molecule 4. Subsequent investigations in this direction have largely been motivated by the experimental demonstration of meta-vs.-para ( $m$-vs.- $p$ ) selectivity in conduction across benzene; for instance, Mayor et al. with their STM-BJ technique demonstrated current flowing through two $m$-linked benzene rings to be two orders of magnitude smaller than that flowing through the $p$ linked analogue 20. Similar conclusions have been drawn also in somewhat more recent experiments on coupled benzene rings 21. More recent experimental efforts have even established such subtle correlations like the effects bond topology and electronegativity of atomic sites can have on the degree and the location of QI features in 
molecular wires 22. It is suggested that a combination of stimuli-response and QI can be an efficient strategy to enhance isomer recognition and conductance switching in single-molecule junctions 23. While conjugation has widely been considered as a main tool to control QI, recent synthetic efforts have shown that the effect can be manipulated through chemical modification of the molecular wire 24]. Exploiting QI for practical purposes requires that decoherence effects are minimal; it is an experimentally established fact that with increase in temperature, destructive interference effects are lost resulting in enhanced conductivity 25].

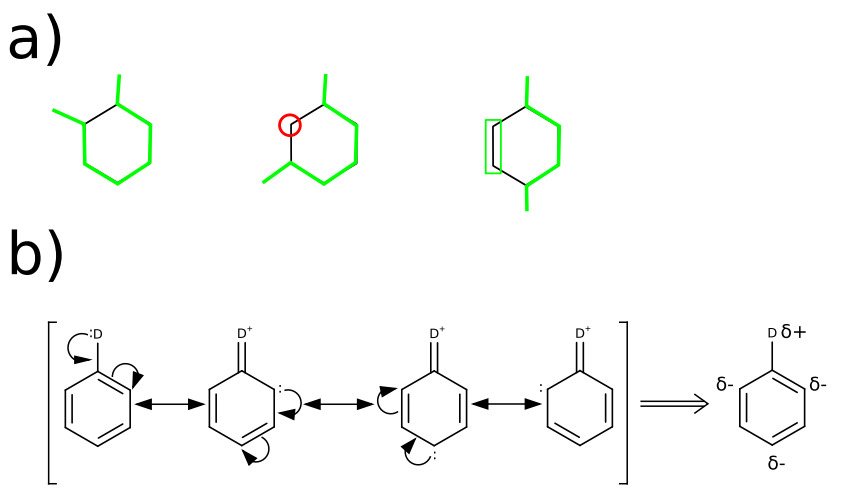

FIG. 1. Prediction of QI features based on molecular graphs and electronic structure. a) Connectivity rules of Markussen et al. based on the longest continuous paths in $o-/ m$ - $/ p$-linked benzenes [26]. An isolated atomic center not located on the path is marked with a red circle in the $m$-linked benzene. b) Curly arrow rules inspired by aromatic resonance stabilization effects 27, 31].

From a theoretical stand point, a number of studies have established qualitative relationships between observed/predicted QI trends with conjugation patterns in hydrocarbons - destructive QI in cross-conjugated molecules and constructive QI in linearly conjugated ones 28 30. In particular, Markussen et al. have presented a set of graphical rules to predict if a molecular structure can lead to QI or not 26]. Accordingly, a molecule will feature destructive QI if the longest continuous path that can be drawn across it connecting both terminals leaves at least one atomic site unconnected (i.e. unpaired) and without a nearest neighbor site. Using this rule, it is straightforward to see why an $m$-linked benzene junction with a single unpaired site will suffer from destructive QI amounting to diminished conductivity (see Fig. 1a). Others have presented a selection rule for QI based on curly arrow diagrams that are traditionally used to diagnose resonance stabilization patterns in $\pi$-electron conjugated systems [27, 31]. Fermi-level destructive QI is noted in a conjugated molecule when curly arrows cannot displace an electron pair from the donor end to at least one of the other sites; Fig. 1 $\mathrm{b}$ showcases how in benzene an electron pair cannot be displaced from the donor end to the $m$ positions. In this context, it may be worthwhile to note that in a theoretical study based on density-matrix propagation based on the tight-binding $\pi$ Hamiltonian, blocking one of the paths in a $p$-linked benzene has resulted in essentially no change in the dynamics of current flow indicating no interference in the $p$-isomer as far as only the $\pi$-channels are concerned 32. The same study showed that an $m$-linked isomer, that had initially shown poor current flow because of phase coherence (or destructive QI) between the current flowing through two paths, has shown much improved dynamics and better rates when one of the paths is blocked.

Interestingly, in contrast to the trends noted for molecular conductivity, Gorczak et al. observed in photoinduced charge transfer (CT) measurements of donorbridge-acceptor (D-B-A) systems faster hole-transfer timescales in cross-conjugated junctions compared to linearly conjugated ones 33. Furthermore, this study has reported D-B-A hole transfer via a $m$-linked biphenyl bridge - with a shorter D-A throughspace distance - to be faster than that via a $p$-linked isomer. The through- $m$ channel also benefits from the involvement of the $\sigma$-typeMOs leading to faster $\mathrm{CT}$ timescales at least in shorter molecular junctions [33, 34. More recent experimental studies have stressed that for a successful rational design of molecular junctions, an understanding of QI effects in $\sigma$-channels is as important as those in $\pi$-channels 35 . The close relation between molecular conduction and Dto-A electron-transfer properties has been discussed by others [36. Meanwhile, somewhat different CT trends have been noted in longer bridge molecules such as crossconjugated xanthone which shows 30 times slower chargeinjection dynamics compared to the linearly-conjugated molecule trans-stilbene. In the former case, it has been argued that cross-conjugation strongly decreases the $\pi$ orbital contribution to D-A electronic coupling so that electron transfer most likely uses the bridge $\sigma$ system as its primary pathway 37 .

It is the purpose of this article to complement continuously evolving chemical intuitions about transport selectivity across isomeric molecular junctions with electron dynamics modeling accounting for many-body coherence and electron correlation effects. To this end, the formally exact formalism of time-dependent configuration interaction (TDCI) 38, 39. has been employed to see electron dynamics in cyanobenzene and in $m / p$-linked benzonitrile thiolate $\left(\mathrm{CN}^{-\mathrm{C}_{6}} \mathrm{H}_{4}\right.$-S- $)$ molecules bonded to a gold $\mathrm{Au}$ ) atom serving as the acceptor terminal. To showcase the applicability of the TDCI methodology to understand electron transfer dynamics selectivity in nonalternant hydrocarbons, we have studied cyanoazulene thiolate molecules $\left(\mathrm{CN}-\mathrm{C}_{10} \mathrm{H}_{6}-\mathrm{S}-\right)$ linked to an $\mathrm{Au}$ atom through four different substitution patterns. 


\section{METHODS}

\section{A. Time-dependent configuration interaction}

Within the scope and restrictions of the BornOppenheimer approximation, any electronic property of a molecule, with a corresponding quantum mechanical operator $\hat{P}$, can be calculated as a function of time once we have the time-dependent wave function $\Psi_{\mathrm{e}}(\mathbf{r}, t)$ obeying the time-dependent Schrödinger equation (TDSE)

$$
i \frac{\partial}{\partial t} \dot{\Psi}_{\mathrm{e}}(\mathbf{r}, t)=\hat{H}_{\mathrm{e}} \Psi_{\mathrm{e}}(\mathbf{r}, t),
$$

where $\Psi_{\mathrm{e}}(\mathbf{r}, t)$ and $\hat{H}_{\mathrm{e}}$ are the electronic wavefunction and the electronic Hamiltonian, respectively. The TDCI approach is formally exact as long as the CI wavefunction is expanded with all possible configuration state functions (CSFs). This incurs very heavy computational requirements and renders all but molecules of the size of water fully tractable. In this study, we have truncated the CI expansion to up to singles and doubles substitution (i.e. CISD):

$$
|\Psi\rangle=c_{0}\left|\Psi_{0}^{\mathrm{HF}}\right\rangle+\sum_{a, r} c_{a}^{r}\left|\Psi_{a}^{r}\right\rangle+\sum_{a<b ; r<s} c_{a, b}^{r, s}\left|\Psi_{a, b}^{r, s}\right\rangle,
$$

where $\left|\Psi_{0}^{\mathrm{HF}}\right\rangle$ is the Hartree-Fock ground state; $\left|\Psi_{a}^{r}\right\rangle$ and $\left|\Psi_{a, b}^{r, s}\right\rangle$ denote singly and doubly substituted Slater determinants, respectively, with $a, b$ going over the indices of occupied spin orbitals while $r, s$ are indices of the unoccupied spin orbitals. The total number of Slater determinants entering the expansion of the CISD wavefunction scales as $\mathcal{O}\left(N_{o} N_{v}\right)$ and $\mathcal{O}\left(N_{o}^{2} N_{v}^{2}\right)\left(N_{o}\right.$ and $N_{v}$ are number of occupied and virtual MOs) for singles and doubles substitution, respectively. Using the fact that the electronic states studied in this work are of singlet-spin symmetry, a more efficient approach is to represent $\Psi(\mathbf{r}, t)$ in the variational space spanned by singlet spin-adapted CSFs 40]:

$$
\begin{aligned}
\left|{ }^{1} \Psi_{a}^{r}\right\rangle= & {\left[\left|\Psi_{\bar{a}}^{\bar{r}}\right\rangle+\left|\Psi_{a}^{r}\right\rangle\right] / \sqrt{2} } \\
\left|{ }^{1} \Psi_{a a}^{r r}\right\rangle= & \left|\Psi_{a \bar{a}}^{r \bar{r}}\right\rangle \\
\left|{ }^{1} \Psi_{a a}^{r s}\right\rangle= & {\left[\left|\Psi_{a \bar{a}}^{r \bar{s}}\right\rangle+\left|\Psi_{a \bar{a}}^{s \bar{r}}\right\rangle\right] / \sqrt{2} } \\
\left|{ }^{1} \Psi_{a b}^{r r}\right\rangle= & {\left[\left|\Psi_{\bar{a} b}^{\bar{r} r}\right\rangle+\left|\Psi_{a \bar{b}}^{r \bar{r}}\right\rangle\right] / \sqrt{2} } \\
\left|{ }^{A} \Psi_{a b}^{r s}\right\rangle= & {\left[2\left|\Psi_{\bar{a} b}^{\bar{r} s}\right\rangle+2\left|\Psi_{\bar{a} \bar{b}}^{\bar{r} \bar{s}}\right\rangle-\left|\Psi_{\bar{a} b}^{\bar{s} r}\right\rangle+\left|\Psi_{\bar{a} b}^{\bar{r} s}\right\rangle+\left|\Psi_{a \bar{b}}^{r \bar{s}}\right\rangle-\right.} \\
& \left.\left|\Psi_{a \bar{b}}^{s \bar{r}}\right\rangle\right] / \sqrt{12} \\
\left|{ }^{B} \Psi_{a b}^{r s}\right\rangle= & {\left[\left|\Psi_{\bar{a} b}^{\bar{s} r}\right\rangle+\left|\Psi_{\bar{a} b}^{\bar{r} s}\right\rangle+\left|\Psi_{a \bar{b}}^{r \bar{s}}\right\rangle+\left|\Psi_{a \bar{b}}^{s \bar{r}}\right\rangle\right] / 2 . }
\end{aligned}
$$

The notation conveys that spin orbital indices with an overline denote beta-spin electrons and those without, alpha-spin electrons. The TDSE is solved as an initial value problem, where qualitative trends in electron dynamics depend on the choice of the initial state.

For the dynamics to result in an efficient CT process, the initial state, $|\Psi(0)\rangle$ must satisfy the following formal criteria: Firstly, since the process being simulated is a field-free evolution with conserved total energy, the initial state must be non-stationary, i.e., formally a wavepacket that is a linear superposition of the electronic energy eigenstates. Secondly, the real-space picture of the initial state must be such that in the neighborhood of the wavepacket's energy, there is a net difference in the density-of-states (DOS) at geometric ends of the molecule. The donor terminal is typically that with an excess density of occupied MOs while that with excess unoccupied MOs mark the acceptor terminal. Experimentally, such an initial electronic configuration can be created in core-hole-clock spectroscopy, where typically, an electron from a main-group atom such as nitrogen is excited to the $\pi^{*}$ MO localized on the CN terminal 41 43. Finite size of the acceptor terminal or finiteness of the states that are localized on the acceptor terminal results in a situation where residual electron density shuttles back and forth between the donor and acceptor ends as noted before in Li-terminated D-B-A molecules [44. In order to stabilize the CT dynamics, electron trapping is essential. This requires that this end is made either of a single transition metal with several unoccupied orbitals or a metal cluster with large number of vacant orbitals. Of utmost importance is also the fact that the bridge region of the molecule must have a large density of vacant MOs serving as the conduction band. Finally, it is important to note that field-free D-B-A CT dynamics is symmetry controlled 42 .

The time-dependent partial charge $q(t)$ on an atom $A$ can be computed by summing over all atomic orbitals (AOs) $\mu$ centered on that atom according to Löwdin's formula

$$
q_{A}(t)=Z_{A}-\sum_{\mu \in A}\left[\mathbf{S}^{1 / 2} \mathbf{P}(t) \mathbf{S}^{1 / 2}\right]_{\mu \mu},
$$

where $\mathbf{S}$ is the overlap matrix in the $\mathrm{AO}$ representation, and $\mathbf{P}(t)$ is the time-dependent reduced charge-density bond-order (CDBO) matrix:

$$
P_{\mu, \nu}(t)=\left\langle\mu\left|\operatorname{Tr}_{2, \ldots, N}\right| \Psi(\mathbf{r}, t)\right\rangle\langle\Psi(\mathbf{r}, t) \mid \nu\rangle .
$$

These matrix elements can be computed for a many-body wavefunction by applying the Slater-Condon rules 40 , 45. With the reduced CDBO matrix, one can plot the three-dimensional electron density as a function of time through

$$
\rho(\mathbf{r}, t)=\sum_{\mu, \nu} \phi_{\mu}(\mathbf{r}) P_{\mu, \nu}(t) \phi_{\nu}(\mathbf{r}),
$$

where $\phi_{\mu}(\mathbf{r})$ is an $\mathrm{AO}$.

\section{B. Computational details}

Minimum energy equilibrium structures of cyanobenzene $\left(\mathrm{C}_{6} \mathrm{H}_{5} \mathrm{CN}\right), p / m$-linked $\mathrm{CN}-\mathrm{C}_{6} \mathrm{H}_{4}-\mathrm{S}$-Au isomers, and $\mathrm{CN}-\mathrm{C}_{10} \mathrm{H}_{6}-\mathrm{S}-\mathrm{Au}$ isomers were optimized at the KS-DFT 


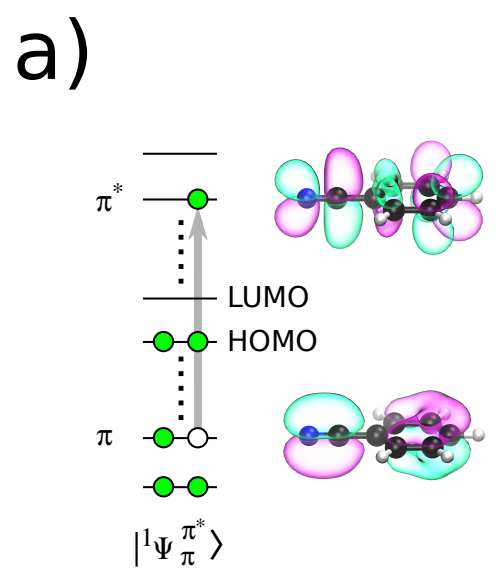

b)

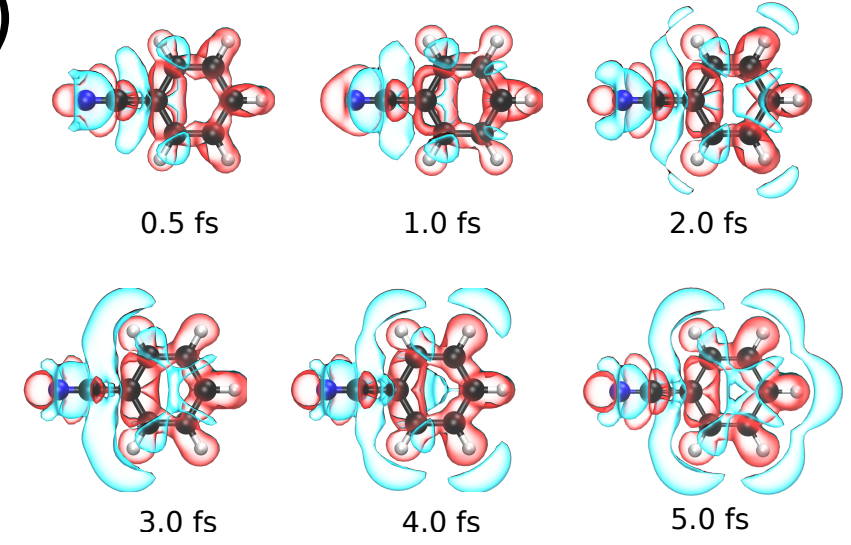

FIG. 2. TDCI wavepacket dynamics of cyanobenzene: a) Creation of a non-stationary electronic state in cyanobenzene by an implicit $\pi \rightarrow \pi^{*}$ single-excitation; plots of the corresponding MOs are shown, b) Electron density difference, $\Delta \rho(\mathbf{r}, t)=$ $\rho(\mathbf{r}, t)-\rho(\mathbf{r}, 0)$, is shown for the first few fs of electron dynamics; blue and red color indicate gain and depletion, respectively. .

level, PBE0[46], using the quantum chemistry package NWCHEM (version 6.6) 47. The split-valence basis set def2-SV $(\mathrm{P})$ 48 containing a polarization function was employed for all atoms. For $\mathrm{Au}$, an effective core potential, ECP60MWB 49, was used to replace the 60 core electrons while the remaining 19 electronsaccounting for the valence configuration $5 s^{2} 5 p^{6} 5 d^{10} 6 s^{1}$ were treated explicitly with the aforementioned basis set. All time-dependent (TD) and time-independent configuration interaction calculations have been performed using locally developed codes 44, 50. The present implementation depends on one- and two-electron molecular integrals along with Hartree-Fock (HF) MOs computed using NWCHEM. Furthermore, all CI calculations have been performed in the framework of spin-adapted CSFs with the CI wavefunction expansion truncated by including up to double substitutions (i.e. CISD). Along with the HF Slater determinant $\left|\Psi_{0}\right\rangle$, we included all possible singly-substituted CSFs, $\left|{ }^{1} \Psi_{a}^{r}\right\rangle$, and we restricted the active space for doubly-substituted CSFs, $\left|{ }^{1} \Psi_{a a}^{r r}\right\rangle,\left|{ }^{1} \Psi_{a a}^{r s}\right\rangle$, $\left|{ }^{1} \Psi_{a b}^{r r}\right\rangle,\left|{ }^{A} \Psi_{a b}^{r s}\right\rangle,\left|{ }^{B} \Psi_{a b}^{r s}\right\rangle$, to $(N=20, M=60)$, where $N$ and $M$ are the number of valence electrons and number of spin orbitals, respectively. For cyanobenzene, $m / p$-CN$\mathrm{C}_{6} \mathrm{H}_{4}$-S-Au isomers, and $\mathrm{CN}_{-} \mathrm{C}_{10} \mathrm{H}_{6}$ - $\mathrm{S}$ - $\mathrm{Au}$ isomers the resulting restricted-active-space-CI (RASCI) 51 wavefunctions contain 22,666, 25,337 and 29,563 CSFs (62,231, 67,573 and 76,025 Slater determinants), respectively. All TD electronic wavepacket propagations were peformed within the fixed-nuclei approximation, which is valid for ultrashort time scales[52. We solved the TDCI equations 39 using the fourth-order RungeKutta method (RK4) with a finite time-step of $\Delta t=$ $0.001 / 4 \pi \mathrm{cRy}=0.024$ attoseconds (as), 1 as $=10^{-18} \mathrm{~s}$.

\section{RESULTS AND DISCUSSIONS}

\section{A. Ultrafast electron dynamics in cyanobenzene}

As a prototype model to illustrate TDCI-based electron dynamics, we begin with the simulation of a fieldfree time-evolution of an electronic wavepacket in the planar molecule $\mathrm{C}_{6} \mathrm{H}_{5} \mathrm{CN}$. Preparation of an initial state for the TDCI dynamics is sketched in Fig. 2a. Since we would like to understand the participation of the out-ofplane $\pi$-type MOs on the benzene fragment - to quantify the relative role of the fragment MOs on the $o-/ m-/ p$ $\mathrm{C}$ atoms - we have chosen a CSF corresponding to the $\pi \rightarrow \pi^{*}$ excitation, where an electron from the occupied $\pi$ MO is excited to the unoccupied $\pi^{*}$ MO. The symmetry of this CSF belongs to the $a^{\prime \prime}$ irreducible representation of the $C_{s}$ point group. Furthermore, $|\Psi(0)\rangle$ features an electronic arrangement that is suitable for CT dynamics satisfying the criteria discussed above. Specifically, at $E=\langle\Psi(0)|\hat{H}| \Psi(0)\rangle$ the projected density of states (PDOS) - corresponding to occupied MOs - is larger on the $\mathrm{CN}$ fragment (the donor terminal) compared to the PDOS on the benzene fragment (acceptor terminal).

Typically, a real-space picture of D-B-A electron dynamics shows electron-injection from the donor terminal to the acceptor end through regions of space localized on the molecular framework suggesting through-bond CT with sufficient directionality. To shed more light on this process, we have plotted the time-dependent electron density, $\rho(\mathbf{r}, t)$, for the first few fs of time-evolution. The dynamics proceeds with a very rapid sub-fs event of refilling of MOs localized at the $\mathrm{CN}$ end by electron density from the benzene fragment. Such a rapid dynamical feature is characteristic of strongly coupled donor and acceptor states arising from good spatial overlap. Further, the energy gap between $\pi$ and $\pi^{*}$ MOs considered for the excitation is $15.6 \mathrm{eV}$, which corresponds to sub-fs oscilla- 


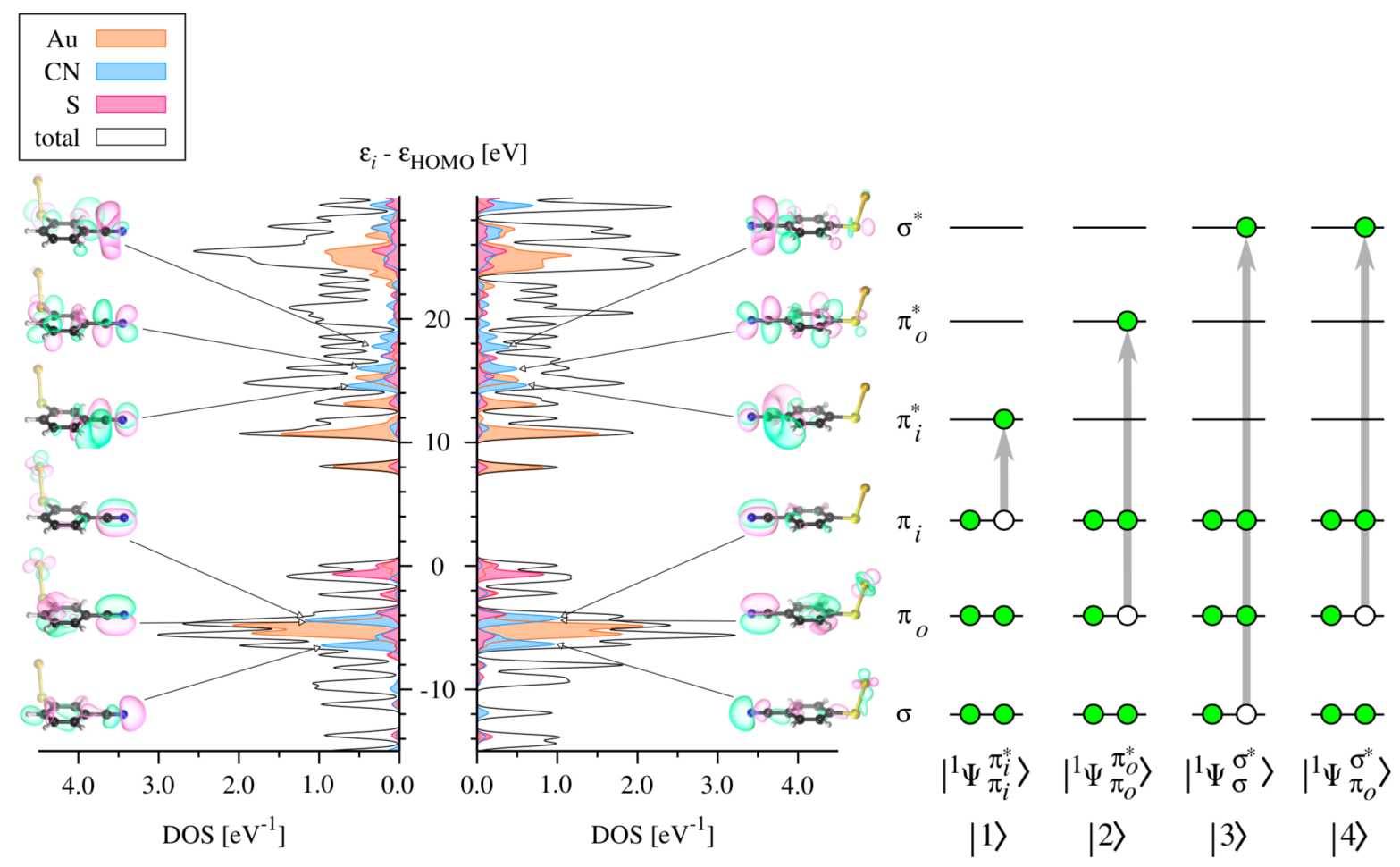

FIG. 3. Electronic structure of $m$ and $p$ isomers of gold-benzonitrile thiolate $\left(\mathrm{CN}_{-} \mathrm{C}_{6} \mathrm{H}_{4}-\mathrm{S}-\mathrm{Au}\right)$. For both molecules, projected density of states (PDOS) are plotted with insets showing selected MOs. Also given are the definitions of various singly substituted CSFs that are used as initial states in TDCI electron dynamics simulations.

tions according to $(\Delta t 0.13 \mathrm{fs})=2.07 /(\Delta E 15.6 \mathrm{eV})$; this is reminiscent of the sub-fs dynamics arising from the ionization of a core electron in nitrosobenzene described by Kuleff et al. [53]. Focussing on Fig. $2 \mathrm{~b}$, by $t=2 \mathrm{fs}$, we note the electron density from the $\mathrm{CN}$ terminal to get injected into the $o$ and $m \mathrm{C}$ sites of benzene. Following brief oscillatory dynamics, by $t=5 \mathrm{fs}$, we also note the $p$ sites of benzene to be populated. Beyond $5 \mathrm{fs}$, as is common in a system with finite DOS localized at the acceptor terminal, the dynamics show recurrences with partial-revival lifetimes of the order of a few fs. Overall, the TDCI electron dynamics of cyanobenzene do not indicate $m$-vs- $p$ selectivity in the population transfer from the $\mathrm{CN}$ group to the $\mathrm{C}$ sites of the benzene. The $\mathrm{CT}$ timescales to populate various sites is strongly dependent on the distance between these sites to the $\mathrm{CN}$ group.

\section{B. Dependence of Electron dynamics on Substitution Patterns}

\section{1. $\quad$-vs-p selectivity in electron dynamics of $\mathrm{CN}-\mathrm{C}_{6} \mathrm{H}_{4}-\mathrm{S}-\mathrm{Au}$}

To induce CT selectivity in TDCI electron dynamics across linked-benzenes - an effect missing in cyanobenzene - we zero-in on the thiolate of benzonitrile bonded to an $\mathrm{Au}$ atom. To this end, we consider both $m$ and $p$ terminated isomers. First of all, inspecting the electronic structure through the DOS near the valence energy, reveals no apparent differences between the $p$ and $m$ isomers (see Fig. 3). Further, fragment-projected DOS reveals all the characteristic MOs localized on D and $\mathrm{A}$ fragments to have very similar energetics across both the isomers. By inspecting the three bonding-type MOs $\sigma(\mathrm{CN}-\mathrm{C}), \pi_{o}(\mathrm{CN}), \pi_{i}(\mathrm{CN})$ - subscripts $o$ and $i$ signify out-of-plane and in-plane w.r.t. the benzene planeand their antibonding counterparts, we notice a flip in the phase of certain MOs of the $m$-isomer compared to the $p$ one.

It is a well known fact based on HMO that $m$ and $p$ substitutions on a benzene ring lead to different phases for selected MOs. In the present work, we note based on HF calculations, that both $\pi_{o} / \pi_{o}^{*}$ MOs show a change in phase when the $m$ link is replaced by a $p$ one. On the other hand, $\pi_{i} / \pi_{i}^{*}$ MOs conserve their phase on both the isomers. Interesting trend is noted for the $\sigma$-type MOs; while the low-energy bonding-type MO shows a phaseflip, the anti-bonding-type MO does not suffer from phase changes. In the next section, we will illustrate how QI features, hence CT selectivity, can be controlled in manyelectron wavepacket dynamics via phase-flip effects in MOs. Having identified the MOs of interest, we consider as initial states for CT dynamics, CSFs formed by exciting an electron from an occupied MO to an unoccupied one (see Fig. 3). It may be worthwhile to note that the 

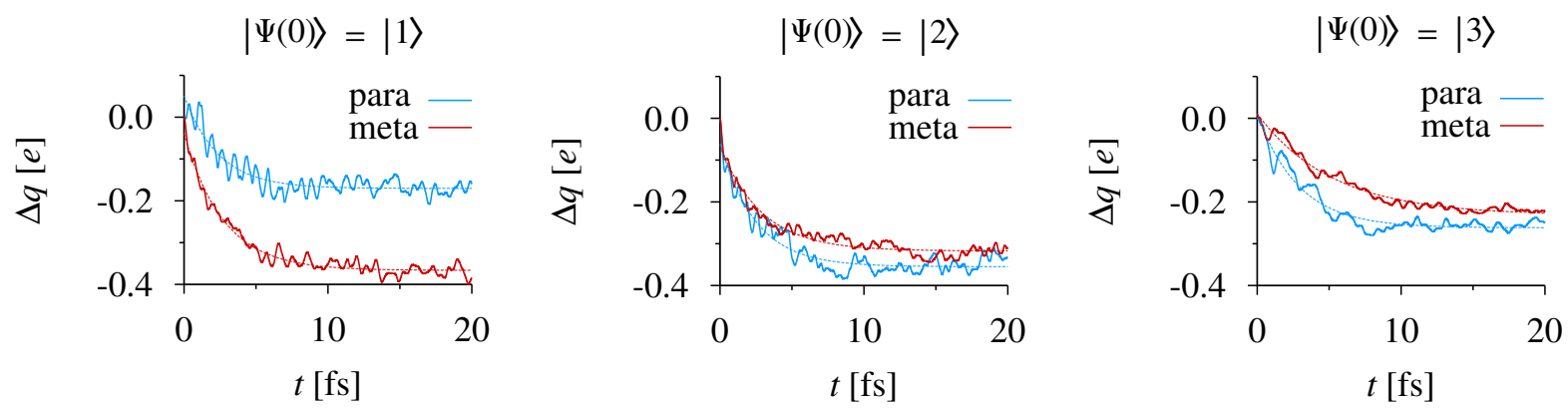

FIG. 4. Time evolution of the partial charge, $q$, on the Au atom in $m$ - and $p$-linked $\mathrm{CN}_{-} \mathrm{C}_{6} \mathrm{H}_{4}-\mathrm{S}$ - $\mathrm{Au}$ for three choices of the initial state, $|\Psi(0)\rangle$ (see Fig. 3 for the definition of initial states). In all cases, net charges are reported after subtracting the value at $t=0$; negative values for $\Delta q$ indicate net flow of electron density from the CN fragment to the metal center.

energies of these CSFs are somewhat higher compared to the HOMO-to-LUMO excitation in these systems. All four CSFs considered here show characteristics suitable to result in stable CT dynamics: i) excess electron density at the $\mathrm{CN}$ donor end compared to the Au acceptor end at $t=0$, ii) availability of several unoccupied orbitals localized on $\mathrm{Au}$ essential for trapping of electron density at the acceptor end for a few fs, and iii) presence of MOs localized on the benzene fragment for conduction.

$\mathrm{CT}$ dynamics in $m$ and $p$ CN- $\mathrm{C}_{6} \mathrm{H}_{4}$-S-Au systems for three different initial states are illustrated in Fig 4 . Time evolution of the partial charge, $q$, on the Au atom reveals selective electron-injection. Overall, one notes the timescales for the events in all the cases to be about 3-4 fs. As the most striking feature, we note CT mediated through the in-plane $\pi(\mathrm{CN})$ orbitals to be more efficient in the $m$ isomer than the $p$ one. However, the same process when mediated either through the out-of-plane $\pi$ or the $\sigma \mathrm{MO}$ is more efficient in the $p$ isomer. This contrasting trend can be understood as follows: All three types of MOs are in resonance with the MOs localized at the $\mathrm{Au}$ acceptor terminal. In addition, the out-of-plane $\pi$ and $\sigma$ MOs are also in resonance with the MOs of same symmetry localized on the benzene fragment (the bridge) amounting to a resonant, through-bond CT process. This situation becomes more apparent through an inspection of the MOs plotted in Fig 3, where one notes the densities of the out-of-plane $\pi$ MOs to be predominantly localized at the donor end, while small but non-vanishing $\mathrm{MO}$ densities localized through the bridge until the $\mathrm{Au}$ end. On the other hand, the in-plane $\pi$ MOs (bonding and antibonding) are strongly localized at the $\mathrm{CN}$ end without mixing of AOs from the benzene ring. Hence, CT mediated by the in-plane $\pi$ MOs is a non-resonant tunneling process - the extent of which diminishes with increase in the distance between the donor and acceptor terminals. Hence, in the $p$-isomer, one notes a drastic drop in the net electron transfer when starting with the $\pi_{i}$-type initial state. The distance dependence of such a non-resonant tunneling process has been demonstrated experimentally [54] for two paracyclophane systems: one where two benzene rings are connected at the para-ends by two methylene units (i.e. 22PCP) and another where the rings are connected by four methylene units in a para fashion (i.e. 44PCP). The inter-ring separation of the shorter and longer molecules are 3 and $4 \AA$, respectively. The CT process in the longer molecule has been found to be 20 times slower than in the shorter PCP [54].

\section{Electron-transfer selectivity in gold-cyanoazulene thiolate isomers}

As a non-trivial case to study electron-transfer selectivity arising from different substitution patterns, we zero-in on cyanoazulene thiolate linked to an $\mathrm{Au}$ atom (see Fig. 5). Following conventional numbering of the $\mathrm{C}$ atoms, we denote the four isomers studied here: $1,3 \mathrm{Az}$, $2,6 \mathrm{Az}, 4,7 \mathrm{Az}$, and $5,7 \mathrm{Az}$, with $\mathrm{Az}$ denoting azulene. Conductivity of these isomers have been the subject of experimental and theoretical investigations $[5561$.

Xia et al. [55] have investigated the conductivity of the aforestated isomers using the STM-BJ technique and NEGF-DFT calculations. This study depended on $\mathrm{Az}$ molecules connected non-covalently to gold junctions through dimethylthiochroman anchor resulting in small numerical values of $G$-in the range $8 \times 10^{-5} G_{0}$ to $32 \times 10^{-5} G_{0}$-when averaged over measurements; the overall trend in conductivity has been concluded as $2,6 \mathrm{Az} \approx 1,3 \mathrm{Az}>4,7 \mathrm{Az}>5,7 \mathrm{Az}$. In the same work, the authors have reported $G$ from NEGF-DFT calculations by setting the Fermi energy, $E_{\mathrm{F}}$, to $-1.5 \mathrm{eV}$ and noted the trend $1,3 \mathrm{Az}>2,6 \mathrm{Az} \approx 4,7 \mathrm{Az}>5,7 \mathrm{Az}$ agreeing semiqualitatively with experimental trends. However, $G$ of $1,3 \mathrm{Az}$ has been noted to drop considerably in an NEGFDFT calculation with $E_{\mathrm{F}}=0 \mathrm{eV}$. Later, Stadler[56] had reiterated the criterion for finite conductance and vanishing destructive QI at $E_{\mathrm{F}}=0 \mathrm{eV}$ to be: all the AOs of the molecular topology should either lie on a continuous path connecting the terminals or lie on a closed loop. As seen in Fig. 5. 1,3Az does not feature unpaired atomic centers indicating the absence of destructive quantum interference. In a separate study, Strange et al. 57 have performed NEGF-DFT calculations for three more isomers, 


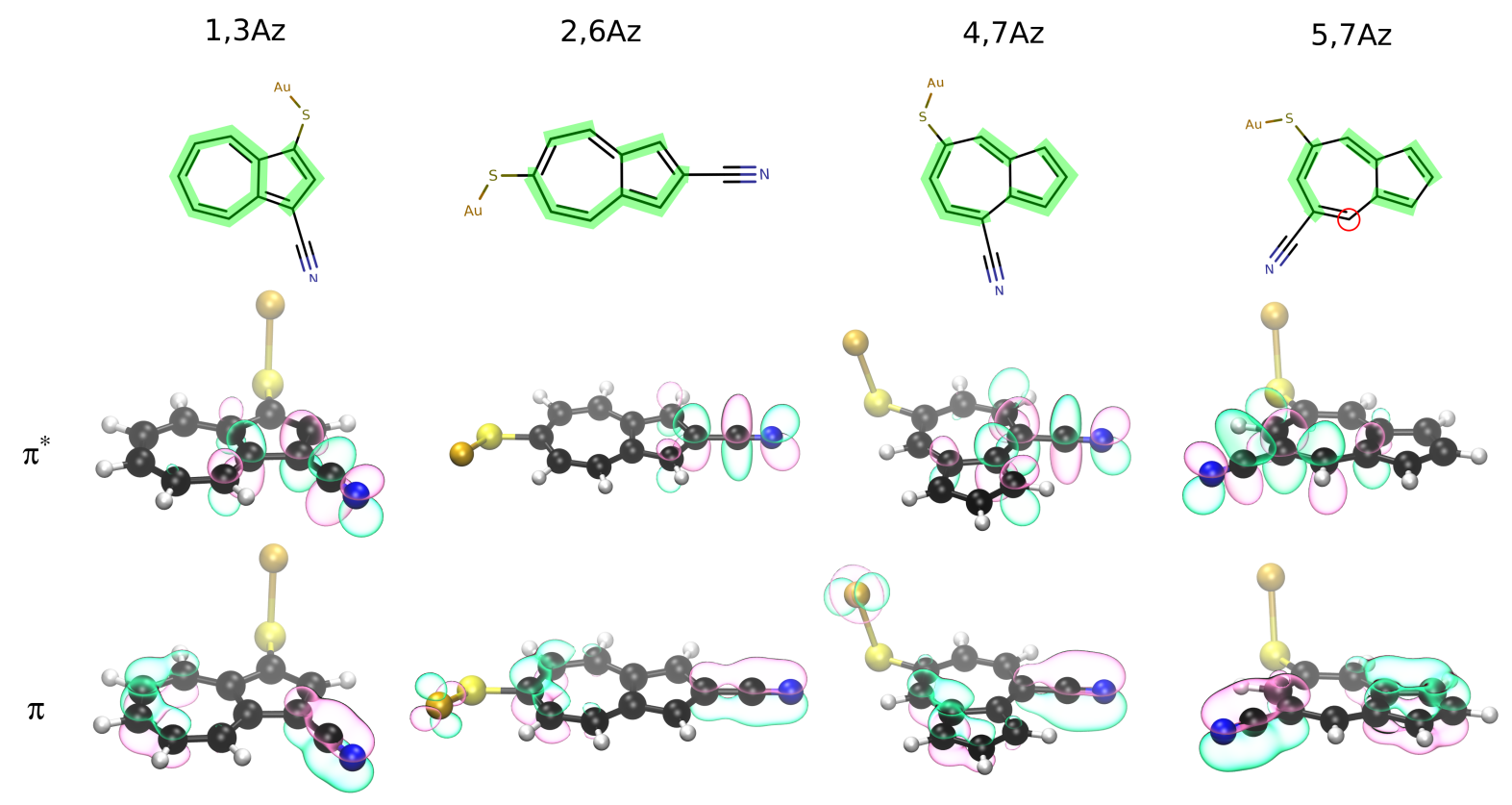

FIG. 5. Isomers of gold-cyanoazulene thiolates with graphical scheme applied for the diagnosis of destructive quantum interference. Also shown are the out-of-plane type $\pi$ and $\pi^{*}$ MOs localized on the CN fragment.

$1,4 \mathrm{Az}, 1,6 \mathrm{Az}$, and $1,8 \mathrm{Az}$ - all of them satisfying the connectivity-based conditions for finite conductance - to exhibit vanishing transmission at $E_{\mathrm{F}}=0 \mathrm{eV}$. This observation strengthened the notion that in these isomers, and in $1,3 \mathrm{Az}$, factors other than interference effects are responsible for a drop in the magnitude of $G$ at $E_{\mathrm{F}}=0 \mathrm{eV}$. In the case of $1,3 \mathrm{Az}$, further clarity emerged from the combined experimental and theoretical work of Schwarz et al. 58 who studied derivatives of $\mathrm{Az}$ that are covalently bonded to the terminals. Compared to the earlier values for a dimethylthiochroman anchor 55 , $2,6 \mathrm{Az}$ and $4,7 \mathrm{Az}$ isomers exhibited better conductance with $G=5 \times 10^{-2} G_{0}$ while $1,3 \mathrm{Az}$ showed $G=1.6 \times 10^{-4} G_{0}$. Overall, the experimental trend in $G$ for the molecules displayed in Fig. 55 at low $T$ and vanishing electronphonon coupling, follows $2,6 \mathrm{Az} \approx 4,7 \mathrm{Az}>1,3 \mathrm{Az}$.

TDCISD CT dynamics of gold cyanoazulene thiolate isomers are shown in Fig. 6. In all cases, the initial state is a CSF corresponding to $\pi_{o} \rightarrow \pi_{o}^{*}$ excitation; the MOs involved are on display in Fig. 5. During the first $10 \mathrm{fs}$ of the dynamics, the time-evolved partial charge on the $\mathrm{Au}$ atom follows the first-order-type relation $\Delta q(t)=\Delta q_{0} \exp (-t / \tau)+\Delta q_{\infty}$. CT parameters estimated via a least squares fitting of $\Delta q(t)$ are collected in

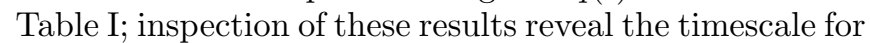
electron migration to lie in the narrow range 2.6-3.0 fs. The most striking qualitative trend as seen in Fig. 5 and Table 1 is that both $2,6 \mathrm{Az}$ and $4,7 \mathrm{Az}$ isomers show larger gain of elecron density at the $\mathrm{Au}$ terminal compared to $1,3 \mathrm{Az}$ and $5,7 \mathrm{Az}$ isomers. The trend in $\Delta q_{\infty}$ can be seen to follow the aforementioned trend noted in experimentally determined $G: 2,6 \mathrm{Az} \approx 4,7 \mathrm{Az}>1,3 \mathrm{Az}>5,7 \mathrm{Az}$.
As noted in a previous TDCI study 44 of Li-terminated cyano-alkenes and -alkynes, a drop in $\Delta q_{\infty}$ is a consequence of either all the MOs involved being fully delocalized from the donor-terminal to the acceptor one, or even when the $\pi$-type-MOs are sufficiently localized on the donor-terminal, truncation in the MO network of suitable symmetry from donor-to-bridge-to-acceptor making CT to proceed only via distance-dependent tunneling. The later mechanism which is on action in $m$-linked benzene also controls the dynamics of $1,3 \mathrm{Az}$ and $5,7 \mathrm{Az}$.

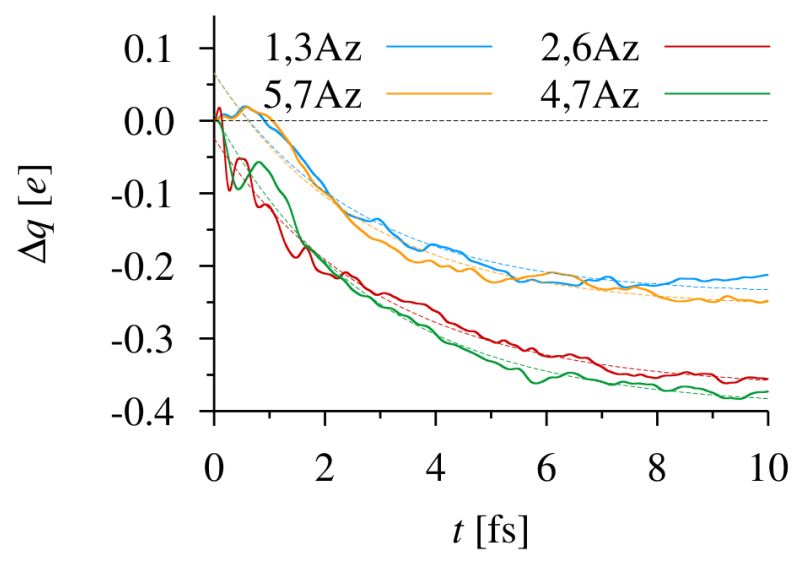

FIG. 6. Time evolution of the partial charge, $q$, on the $\mathrm{Au}$ atom in $\mathrm{CN}_{-} \mathrm{C}_{10} \mathrm{H}_{6}-\mathrm{S}-\mathrm{Au}$ isomers; net charge $(\Delta q)$ is reported after subtracting the value at $t=0$; negative values indicate flow of electron density from the CN fragment to the metal center. In all cases, the initial state, $|\Psi(0)\rangle$ is created by exciting an electron from the $\pi_{o}(\mathrm{CN}) \mathrm{MO}$ to the $\pi_{o}^{*}(\mathrm{CN}) \mathrm{MO}$. 
TABLE I. Charge-transfer parameters for gold-cyanoazulene thiolate isomers obtained by fitting the time-evolution of the net-electron gain, $\Delta q$, at the gold terminal to $\Delta q(t)=$ $\Delta q_{0} \exp (-t / \tau)+\Delta q_{\infty}$. Net charges are reported in $e$ and the timescale is in fs.

\begin{tabular}{lccl}
\hline Molecule & $\Delta q_{0}$ & $\Delta q_{\infty}$ & $\tau$ \\
\hline $1,3 \mathrm{Az}$ & 0.06 & -0.24 & 2.62 \\
$2,6 \mathrm{Az}$ & -0.02 & -0.37 & 3.03 \\
$4,7 \mathrm{Az}$ & 0.01 & -0.40 & 2.88 \\
$5,7 \mathrm{Az}$ & 0.07 & -0.26 & 2.68 \\
\hline
\end{tabular}

\section{Quantum interference via linear superposition of many-body wavefunctions}

Tsuji et al. 29, 60] have shown and discussed with great clarity that in conductivity calculations based on the HMO model Hamiltonian, QI can be predicted for a pair of atoms $\mu$ and $\nu$ using the zeroth-order Green's function

$$
G_{\mu \nu}^{0}\left(E_{\mathrm{F}}\right)=\sum_{k \in \mathrm{MOs}} \frac{C_{\mu k} C_{\nu k}^{*}}{E_{\mathrm{F}}-E_{k}+i \eta},
$$

where $E_{\mathrm{F}}$ is the Fermi energy, $E_{k}$ is the energy of the $k$-th $\mathrm{MO}$ and $\eta$ is a small positive number. The molecular junction features destructive QI when $G_{\mu \nu}^{0}\left(E_{\mathrm{F}}\right)$ vanishes for $E_{\mathrm{F}}=0$. For any pair of MOs (say $i$-th $\mathrm{MO}$ is $\pi$ and $j$-th is $\pi^{*}$ ), with an assumption that $E_{\mathrm{F}}$ lies between the energy levels of the frontier MOs (FMOs), i.e., $E_{i}<0$ and $E_{j}>0, G_{\mu \nu}^{0}\left(E_{\mathrm{F}}\right)$ diminishes when $\operatorname{sgn}\left(C_{\mu i} C_{\nu i}^{*}\right)=\operatorname{sgn}\left(C_{\mu j} C_{\nu j}^{*}\right)$, where $\operatorname{sgn}()$ is the sign function. On the other hand, for the situation $\operatorname{sgn}\left(C_{\mu i} C_{\nu i}^{*}\right)=$ $-\operatorname{sgn}\left(C_{\mu j} C_{\nu j}^{*}\right)$, the contributions to the Green's function from FMOs $i$ and $j$ add up amounting to better transmission. Following this argument, it is rather straightforward to see how the $m$-vs.- $p$ selectivity can be interpreted as arising from the phase-flip of one of the FMOs in the $m$ isomer compared to the $p$ counterpart. Koga et al. 15. have shown using DFT-NEGF calculations and symmetry arguments how the aforestated selectivity is preserved when coupling the benzene bridge to $\pi$-acceptor anchor groups.

To realize QI in real-time electron dynamics simulations, we consider initial states that are linear superpositions of CSFs. When following the time evolution of any quantum mechanical observable, interference effects arise from the off-diagonal (or coherence) terms in the expectation value. If the initial state is given by the symmetric linear combination $|\Psi(0)\rangle=[|1\rangle+|2\rangle] / \sqrt{2}$, the expectation value of an observable $O$ is given by $<\hat{O}>=<\hat{O}>_{\text {ave. }}+<\hat{O}>_{\text {int. }}$, where the first term denotes averaging over both states, $<\hat{O}>_{\text {ave. }}=$ $\left[O_{11}+O_{22}\right] / 2$, while the second term arises due to interference, $\langle\hat{O}\rangle_{\text {int. }}=\left[O_{12}+O_{21}\right] / 2=\mathcal{R} e\left[O_{12}\right]$.

To form initial states that are linear superpositions, we consider three CSFs (Fig 3 ): $|2\rangle$ (corresponding to $\pi_{o} \rightarrow \pi_{o}^{*}$ excitation), $|3\rangle$ (corresponding to $\sigma \rightarrow \sigma^{*}$ excitation) and $|4\rangle$ (corresponding to $\pi_{o} \rightarrow \sigma^{*}$ excita- tion). Fig 7 features time-dependent partial charge on the $\mathrm{Au}$ acceptor terminal for two choices of initial states: $|\Psi(0)\rangle=[|2\rangle+|3\rangle] / \sqrt{2}$ and $|\Psi(0)\rangle=[|2\rangle+|4\rangle] / \sqrt{2}$. Starting with the first option, we see that both in the $m$ and $p$ isomers, CT dynamics follow the average of the dynamics exhibited separately by states $|2\rangle$ and $|3\rangle$ indicating vanishing contributions from the interference terms. This can be understood through a consideration of the Slater-Condon rules to evaluate matrix elements for a one-electron operator, $\mathcal{O}_{1}$ 40. Accordingly, at $t=0 \mathrm{fs}$, direct terms contributing to $q(t)$ can be determined as $\left\langle\Psi_{a}^{r}\left|\mathcal{O}_{1}\right| \Psi_{a}^{r}\right\rangle=\sum_{c}^{N}\left\langle c\left|\mathcal{O}_{1}\right| c\right\rangle-\left\langle a\left|\mathcal{O}_{1}\right| a\right\rangle+\left\langle r\left|\mathcal{O}_{1}\right| r\right\rangle$. As for the coherence terms, contributions arise from the matrix elements of the form $\left\langle\Psi_{a}^{r}\left|\mathcal{O}_{1}\right| \Psi_{b}^{s}\right\rangle$. This matrix element vanishes according to the Slater-Condon rules 40, when $a \neq b ; r \neq s$. Non-vanishing contributions to interference terms arise only when $a=b ; r \neq s ;\left\langle\Psi_{a}^{r}\left|\mathcal{O}_{1}\right| \Psi_{a}^{s}\right\rangle=$ $\left\langle r\left|\mathcal{O}_{1}\right| s\right\rangle$ or $a \neq b ; r=s ;\left\langle\Psi_{a}^{r}\left|\mathcal{O}_{1}\right| \Psi_{b}^{r}\right\rangle=-\left\langle b\left|\mathcal{O}_{1}\right| a\right\rangle$. While inspecting the time-evolution of $\Delta q$ at the Au terminal, starting with the superposed initial state $|\Psi(0)\rangle=$ $[|2\rangle+|4\rangle] / \sqrt{2}$, we note in the case of the $p$ isomer, superior net CT compared to the average of the dynamics exhibited separately by $|2\rangle$ and $|4\rangle$. In contrast, for the $m$ isomer, $\Delta q$ drops noticeably compared to the average dynamics. This trend suggests that QI contributions in real-time dynamics with many-electron wavefunctions can feature both constructive and destructive QI effects depending on the sign of the coherence contributions to the time-dependent expectation values.

\section{CONCLUSIONS}

In summary, following the electron dynamics in realtime with the TDCI method offers an exact, all-electron and many-body picture of ultrafast electron dynamics in D-B-A systems with linked-benzene bridges. The dynamics is sensitive to the choice of the initial state. In cyanobenzene, when starting with an initial state created by $\pi \rightarrow \pi^{*}$ excitation, electron density is injected into the benzene fragment within the first 5 fs. During the initial part of time-evolution, the net CT is maximal at the $o$ position. By $t=4$ and $t=5 \mathrm{fs}$, electron density reaches $m$ - and $p$-sites, respectively, with essentially no preference for one site over the other beyond that demanded by distance. The CT process is oscillatory with very short timescales typical of wavepacket evolution in finite systems. Attaching the benzene molecule to an acceptor terminal linked at $m$ or $p$ positions stabilizes the CT process and delays wavepacket revival. Dynamics involving CN out-of-plane $\pi$ or $\sigma$ MOs show enhanced CT in the $p$ isomer compared to the $m$ one. On the other hand, dynamics along the in-plane $\pi$ channel shows counterintuitive selectivity where the CT in the $m$ isomer is more efficient than the $p$ isomer due to non-resonant tunneling that drops rapidly with increase in the distance between $\mathrm{D}$ and $\mathrm{A}$ terminals. TDCI dynamics distinguish $1,3 \mathrm{Az}, 2,6 \mathrm{Az}, 4,7 \mathrm{Az}$, and $5,7 \mathrm{Az}$ isomeric bridges into 


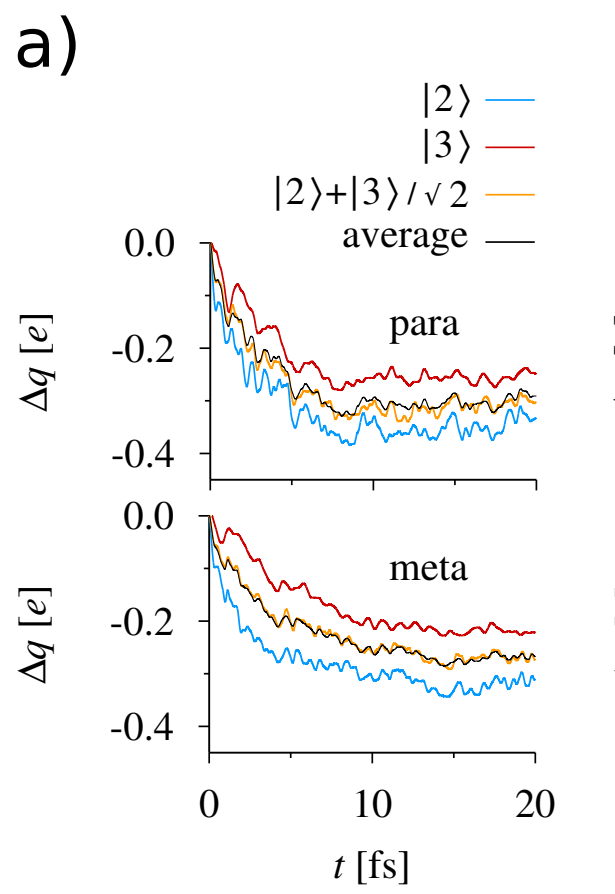

b)

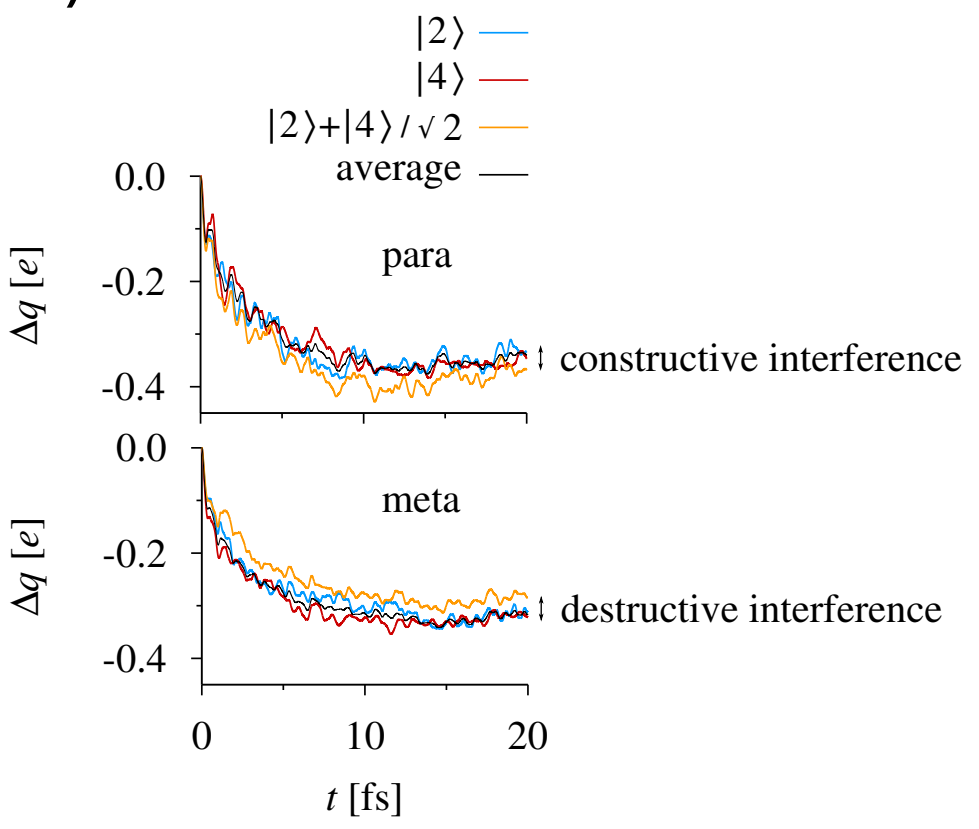

FIG. 7. Time evolution of net-electron gain, $\Delta q$, at the $\mathrm{Au}$ atom in $m$ - and $p$-linked $\mathrm{CN}-\mathrm{C}_{6} \mathrm{H}_{4}-\mathrm{S}-\mathrm{Au}$. Results are shown for two choices of initial states that are coherent superpositions: a) Dynamics of $[|2\rangle+|3\rangle] / 2$ coincides with the average of the values from the individual states $|2\rangle$ and $|3\rangle$ indicating negligible interference effects. b) $\Delta q(t)$ of the superposed state showing enhanced electron migration for the $p$ isomer and a suppressed migration for the $m$ isomer compared to the average of the values from the individual states $|2\rangle$ and $|4\rangle$.

two classes: those with conjugated $\pi$-type MO network on donor, bridge and acceptor sites; those where the $\pi$ type MO network is disrupted. $2,6 \mathrm{Az}$ and $4,7 \mathrm{Az}$ isomers belong to the former class permitting large net $\mathrm{CT}$ while $1,3 \mathrm{Az}$ and $5,7 \mathrm{Az}$ isomers belonging to the latter case exhibit small net CT arising from non-resonant tunneling. This observation clarifies the poor conductivity of the $1,3 \mathrm{Az}$ isomer, which has been noted as a pathological case in transport studies because of the sensitivity of its $G$ with the change in the anchor group coupling azulene to metal terminals 5558 .

Compared to the Green's function and density matrix formalisms, where QI features appear due to cancellation of phases in different paths, in the many body approach presented here, we see QI appearing due to phase cancellations of many-electron wavefunctions that are spread over all possible paths. The TDCI formalism can be adapted to model finite-bias conductance to study metalmolecule-metal junctions. Such a formalism based on localized density constraints to create a chemical potential bias has been developed in the framework of RT-DFT and has been shown to give $I-V$ curves of a molecular wire in agreement with Green's function calculations 62. This procedure when used with a many-body formalism like TDCI, besides providing quantitative state-selective details that are not accessible in DFT-based Green's function calculations, can also address ambiguities that arise in single-determinant electron dynamics 63. Modifications can also be made to the choice of the junction contacts by replacing the thiolate group with an amine group that has shown to result in more reproducible conductance 64.

\section{ACKNOWLEDGMENTS}

The author gratefully thanks Ravi Venkatramani and his group members for countless discussions on chargetransfer phenomena, Reviewer-2 for thought-provoking comments, and Salini Senthil for assistance with the manuscript revision. This project was funded by intramural funds at TIFR Hyderabad from the Department of Atomic Energy (DAE). All calculations have been performed using the Helios computer cluster, which is an integral part of the MolDis Big Data facility, TIFR Hyderabad (https://moldis.tifrh.res.in/).
[1] A. Aviram and M. A. Ratner, Chem. Phys. Lett. 29, 277 (1974).
[2] M. A. Reed and J. M. Tour, Sci. Am. 282, 86 (2000). 
[3] J. R. Heath and M. A. Ratner, Physics Today 56, 43 (2003).

[4] A. Nitzan and M. A. Ratner, Science 300, 1384 (2003).

[5] C. Joachim, J. Gimzewski, and A. Aviram, Nature 408, $541(2000)$.

[6] T. A. Su, M. Neupane, M. L. Steigerwald, L. Venkataraman, and C. Nuckolls, Nat. Rev. Mater 1, 16002 (2016).

[7] H. Weber, J. Reichert, F. Weigend, R. Ochs, D. Beckmann, M. Mayor, R. v. Ahlrichs, and H. Löhneysen, Chem. Phys. 281, 113 (2002).

[8] S. V. Aradhya, J. S. Meisner, M. Krikorian, S. Ahn, R. Parameswaran, M. L. Steigerwald, C. Nuckolls, and L. Venkataraman, Nano Lett. 12, 1643 (2012).

[9] L. Venkataraman, J. E. Klare, C. Nuckolls, M. S. Hybertsen, and M. L. Steigerwald, Nature 442, 904 (2006).

[10] B. Xu and N. J. Tao, science 301, 1221 (2003).

[11] G. Cuniberti, G. Fagas, and K. Richter, in Introducing Molecular Electronics: A Brief Overview (Springer, 2006).

[12] S. Datta, Quantum transport: atom to transistor (Cambridge university press, 2005).

[13] K. G. Pedersen, A. Borges, P. Hedegård, G. C. Solomon, and M. Strange, J. Phys. Chem. C 119, 26919 (2015).

[14] M. Brandbyge, J.-L. Mozos, P. Ordejón, J. Taylor, and K. Stokbro, Phys. Rev. B 65, 165401 (2002).

[15] J. Koga, Y. Tsuji, and K. Yoshizawa, J. Phys. Chem. C 116, 20607 (2012).

[16] M. Büttiker, Physica Scripta 1986, 82 (1986).

[17] P. Sautet and C. Joachim, Chem. Phys. Lett. 153, 511 (1988).

[18] S.-H. Ke, W. Yang, and H. U. Baranger, Nano Lett. 8, 3257 (2008).

[19] S. Datta, Electronic Transport In Mesoscopic Systems (Cambridge University Press, 1997).

[20] M. Mayor, H. B. Weber, J. Reichert, M. Elbing, C. von Haenisch, D. Beckmann, and M. Fischer, Angew. Chem. Int. Ed. 42, 5834 (2003).

[21] C. R. Arroyo, S. Tarkuc, R. Frisenda, J. S. Seldenthuis, C. H. Woerde, R. Eelkema, F. C. Grozema, and H. S. Van Der Zant, Angew. Chem. Int. Ed. 52, 3152 (2013).

[22] Y. Zhang, G. Ye, S. Soni, X. Qiu, T. L. Krijger, H. T. Jonkman, M. Carlotti, E. Sauter, M. Zharnikov, and R. C. Chiechi, Chem. Sci. 9, 4414 (2018).

[23] Y.-P. Zhang, L.-C. Chen, Z.-Q. Zhang, J.-J. Cao, C. Tang, J. Liu, L.-L. Duan, Y. Huo, X. Shao, W. Hong, et al., J. Am. Chem. Soc. 140, 6531 (2018).

[24] S. Naghibi, A. K. Ismael, A. Vezzoli, M. K. AlKhaykanee, X. Zheng, I. M. Grace, D. Bethell, S. J. Higgins, C. J. Lambert, and R. J. Nichols, J. Phys. Chem. Lett. 10, 6419 (2019).

[25] S. Ballmann, R. Härtle, P. B. Coto, M. Elbing, M. Mayor, M. R. Bryce, M. Thoss, and H. B. Weber, Phys. Rev. Lett. 109, 056801 (2012).

[26] T. Markussen, R. Stadler, and K. S. Thygesen, Nano Lett. 10, 4260 (2010).

[27] T. Stuyver, S. Fias, F. De Proft, and P. Geerlings, J. Phys. Chem. C 119, 26390 (2015).

[28] M. G. Reuter and T. Hansen, J. Chem. Phys. 141 (2014).

[29] Y. Tsuji, E. Estrada, R. Movassagh, and R. Hoffmann, Chem. Rev. 118, 4887 (2018).

[30] D. Nozaki, A. Lücke, and W. G. Schmidt, J. Phys. Chem. Lett. 8, 727 (2017).

[31] H. Hosoya, Current Organic Chemistry 19, 293 (2015).
[32] S. Chen, Y. Zhang, S. Koo, H. Tian, C. Yam, G. Chen, and M. A. Ratner, J. Phys. Chem. Lett. 5, 2748 (2014).

[33] N. Gorczak, N. Renaud, S. Tarkuç, A. J. Houtepen, R. Eelkema, L. D. Siebbeles, and F. C. Grozema, Chem. Sci. 6, 4196 (2015).

[34] A. Borges, E.-D. Fung, F. Ng, L. Venkataraman, and G. C. Solomon, J. Phys. Chem. Lett. 7, 4825 (2016).

[35] M. H. Garner, H. Li, Y. Chen, T. A. Su, Z. Shangguan, D. W. Paley, T. Liu, F. Ng, H. Li, S. Xiao, et al., Nature 558, 415 (2018).

[36] A. Nitzan, J. Phys. Chem. A 105, 2677 (2001).

[37] A. B. Ricks, G. C. Solomon, M. T. Colvin, A. M. Scott, K. Chen, M. A. Ratner, and M. R. Wasielewski, J. Am. Chem. Soc. 132, 15427 (2010).

[38] T. Klamroth and M. Nest, Phys. Chem. Chem. Phys. 11, 349 (2009).

[39] P. Krause, T. Klamroth, and P. Saalfrank, J. Chem. Phys. 123, 074105 (2005).

[40] A. Szabo and N. S. Ostlund, Modern Quantum Chemistry: Introduction to Advanced Electronic Structure Theory (Dover Publications, New York, 1996).

[41] H. Hamoudi, S. Neppl, P. Kao, B. Schüpbach, P. Feulner, A. Terfort, D. Allara, and M. Zharnikov, Phys. Rev. Lett. 107, 027801 (2011).

[42] F. Blobner, P. B. Coto, F. Allegretti, M. Bockstedte, O. Rubio-Pons, H. Wang, D. L. Allara, M. Zharnikov, M. Thoss, and P. Feulner, J. Phys. Chem. Lett. 3, 436 (2012).

[43] A. Föhlisch, P. Feulner, F. Hennies, A. Fink, D. Menzel, D. Sanchez-Portal, P. Echenique, and W. Wurth, Nature 436, 373 (2005).

[44] R. Ramakrishnan, S. Raghunathan, and M. Nest, Chem. Phys. 420, 44 (2013).

[45] I. S. Ulusoy and M. Nest, J. Am. Chem. Soc. 133, 20230 (2011).

[46] C. Adamo and V. Barone, J. Chem. Phys. 110, 6158 (1999).

[47] M. Valiev, E. J. Bylaska, N. Govind, K. Kowalski, T. P. Straatsma, H. J. Van Dam, D. Wang, J. Nieplocha, E. Apra, T. L. Windus, et al., Comput. Phys. Comm. 181, 1477 (2010).

[48] F. Weigend and R. Ahlrichs, Phys. Chem. Chem. Phys. 7, 3297 (2005).

[49] D. Andrae, U. Haeussermann, M. Dolg, H. Stoll, and H. Preuss, Theor. Chim. Acta 77, 123 (1990).

[50] R. Ramakrishnan and M. Nest, Chem. Phys. 446, 24 (2015).

[51] D. Hochstuhl and M. Bonitz, Phys. Rev. A 86, 053424 (2012).

[52] I. S. Ulusoy and M. Nest, J. Phys. Chem. A 116, 11107 (2012).

[53] A. I. Kuleff, N. V. Kryzhevoi, M. Pernpointner, and L. S. Cederbaum, Phys. Rev. Lett. 117, 093002 (2016).

[54] A. Batra, J. S. Meisner, P. Darancet, Q. Chen, M. L. Steigerwald, C. Nuckolls, and L. Venkataraman, Faraday Discuss. 174, 79 (2014).

[55] J. Xia, B. Capozzi, S. Wei, M. Strange, A. Batra, J. R. Moreno, R. J. Amir, E. Amir, G. C. Solomon, L. Venkataraman, et al., Nano Lett. 14, 2941 (2014).

[56] R. Stadler, Nano Lett. 15, 7175 (2015).

[57] M. Strange, G. Solomon, L. Venkataraman, and L. Campos, Nano letters 15, 7177 (2015).

[58] F. Schwarz, M. Koch, G. Kastlunger, H. Berke, R. Stadler, K. Venkatesan, and E. Lörtscher, Ange- 
wandte Chemie International Edition 55, 11781 (2016).

[59] X. Zhao, V. Geskin, and R. Stadler, J. Chem. Phys. 146, 092308 (2017).

[60] Y. Tsuji and K. Yoshizawa, J. Phys. Chem. C 121, 9621 (2017).

[61] T. Waechter, K. J. Scheetz, A. D. Spaeth, M. V. Barybin, and M. Zharnikov, The Journal of Physical Chemistry C 121, 13777 (2017).
[62] C.-L. Cheng, J. S. Evans, and T. Van Voorhis, Phys. Rev. B 74, 155112 (2006).

[63] R. Ramakrishnan and M. Nest, Phys. Rev. A 85, 054501 (2012).

[64] L. Venkataraman, J. E. Klare, I. W. Tam, C. Nuckolls, M. S. Hybertsen, and M. L. Steigerwald, Nano Lett. 6, 458 (2006). 